\title{
Identification of 4-methoxythieno[2,3-d]pyrimidines as FGFR1 Inhibitors
}

\author{
I. M. Kotey, M. V. Protopopov, S. A. Starosyla, A. O. Balanda, L. V. Pletnova, \\ A. O. Prykhod'ko, V. G. Bdzhola, S. M. Yarmoluk \\ Institute of Molecular Biology and Genetics, NAS of Ukraine \\ 150, Akademika Zabolotnoho Str., Kyiv, Ukraine, 03143 \\ sergiy@yarmoluk.org.ua
}

\begin{abstract}
Aim. To identify novel FGFR1 inhibitors using virtual screening approach. Methods. We used methods of organic synthesis, molecular docking via the Autodock 4.2.6 program package and in vitro biochemical tests with $\gamma_{-3}{ }^{32} \mathrm{P}$. Results. In vitro experiments showed that 9 of 23 tested compounds possess inhibitory activity against FGFR1 with $\mathrm{IC}_{50}$ values in the range from 0.9 to $5.6 \mu \mathrm{M}$. Conclusions. Nine FGFR1 inhibitors were developed. The mode of compounds binding with the ATP-acceptor site was determined using molecular docking methods and the dependence of the compounds' activity on the substituents $\mathrm{R}^{1}, \mathrm{R}^{4}$ and $\mathrm{R}^{5}$ was evaluated.
\end{abstract}

Keywords: Fibroblast growth factor receptor 1, molecular docking, organic synthesis, in vitro testing, 4-methoxythieno[2,3-d]pyrimidin.

\section{Introduction}

Fibroblast growth factor receptor 1 (FGFR1) is a receptor tyrosine kinase. Normally this enzyme is involved in tissue repair, hematopoiesis, angiogenesis and embryonic development. However, due to the FGFR role in regulating cell growth and survival, amplification and/or mutation of this protein kinase drives a number of cancer types, including gastric cancer, multiple myeloma, breast cancer and nonsmall cell lung cancer (NSCLC). Such inhibitors of FGFR1 as AZD4547, E3810, G141 and E7090 showed anticancer potential and now are in clinical trials. This means that FGFR1 is an appealing target for the drug development [1-4].

The main aim of this work was the search for new FGFR1 inhibitors among 4-methoxythieno[2,3-d]pyrimidine derivatives. Earlier using molecular docking of the collection of the Department of medicinal chemistry (Institute of molecular biology and genetics, NAS of Ukraine) we identified several classes of FGFR1 inhibitors, such as N-phenylnaphthostyril-1-sulfonamides [5], N-phenylthieno[2,3-d]pyrimidin-4-amines [6], quinazolines [7], oxindoles [8], (1H-benzoimidazol-2-yl)-

(C) 2019 I. M. Kotey et al.; Published by the Institute of Molecular Biology and Genetics, NAS of Ukraine on behalf of Biopolymers and Cell. This is an Open Access article distributed under the terms of the Creative Commons Attribution License (http://creativecommons.org/licenses/by/4.0/), which permits unrestricted reuse, distribution, and reproduction in any medium, provided the original work is properly cited 
phenyl-1,2-dihydro-pyrrol-3-ones [9] and flavones [10].

\section{Materials and methods}

\section{Molecular docking}

Preparation of ligands and receptor molecules. Autodock 4.2.6 programs package was used for the receptor-based flexible docking [11]. Ligands were prepared by Vega ZZ (command line) [12] and MGL Tools 1.5.6 [11]. The incoming formats of receptor and ligands data were converted into PDBQT-format with Vega $\mathrm{ZZ}$ using AUTODOCK force field. This format contains the coordinates of the atoms and partial charges. Hydrogen atoms were removed from nonpolar atoms. The receptor was prepared using MGL Tools and AutoGrid [11].

We have used docking parameters reported earlier [13].

Flexible docking. The catalytic subunit of protein kinase FGFR1 complex with inhibitor (PDB code 3GQI) was used for molecular docking via Autodock [14]. Water molecules, ions, and ligands were removed from the PDB file.

\section{Visual analysis}

A visual analysis of the results of molecular docking (interaction of compounds with the amino acid residues of FGFR1 ATP-binding site) was carried out in the Discovery Studio Visualizer 4.0 (http://accelrys.com/).

\section{In vitro testing}

The FGFR1 kinase assays with recombinant cytoplasmic domain of the FGFR1 tyrosine kinase (Millipore, Cat. N. 14-582) were performed in a total volume of $30 \mu \mathrm{l}$ containing $10 \mathrm{mM}$
MOPS ( $\mathrm{pH} 7.2$ ), $0.1 \mathrm{mM}$ sodium orthovanadate, $0.2 \mathrm{mM}$ EDTA, $0.002 \%$ Brij 35, $0.2 \mathrm{mg} / \mathrm{ml}$ BSA, $0.02 \% \beta$-mercaptoethanol, $250 \mu \mathrm{M}$ of peptide substrate (KKKSPGEYVNIEFG, GenScript), various concentrations of inhibitor dissolved in DMSO (final DMSO concentration in probe less than $1 \%$ ) and $10 \mathrm{mU}$ of enzyme. The reaction was initiated by the addition of ATP (50 $\mu \mathrm{M}$ ATP, $25 \mathrm{mM} \mathrm{MgAc}$ containing $0.1 \mu \mathrm{Ci}$ of $\left[\gamma^{32} \mathrm{P}\right]$ ATP per probe) and samples incubated at $30^{\circ} \mathrm{C}$ for $25 \mathrm{~min}$. The reaction was terminated by the addition of $5 \%$ phosphoric acid and the precipitation of material onto phosphocellulose filters "Whatman P81". Filters were washed three times with $0.75 \%$ phosphoric acid and the incorporation of $\left[{ }^{32} \mathrm{P}\right]$ into the peptide substrate was determined by counting the radioactivity retained on the filters in a PerkinElmer scintillation counter. As negative control an equal volume of dimethyl sulfoxide (DMSO) was added to the reaction mixture. Inhibition percentage was calculated as a ratio of the substrate-incorporated radioactivity in the presence of inhibitor to the radioactivity incorporated in control reactions, i.e. in the absence of inhibitor. The concentration of compound that inhibited enzymatic activity by $50 \%$ (IC50) was determined graphically.

\section{Chemistry}

The thieno[2,3-d]pyrimidine derivatives were synthesized from corresponding carbonyl compounds according to Scheme 1. Initially substituted 2-amino-3-carbethoxythiofenes (2) were obtained by Gewald reaction (in case of acetophenone [the] synthesis was carried out in two stages: firstly ethyl cyanoacetate was condensed with the corresponding acetophenone and then the product was cyclized with 
sulfur) (method 1). Then thienopyrimidinones (3) were obtained by condensation of 2-amino3 -carbethoxythiofenes with formamide (method 2). The various 4-chloro-thieno[2,3-d]pyrimidines (4) were synthesized after interaction of thienopyrimidinones with $\mathrm{PCl}_{5}$ (method 3 ) and used as a building block for further formation of combinatorial rows. The final compounds were obtained by chlorine substitution in previous molecules with corresponding phenols (method 4a) or protected aminophenols (method 4b).

\section{Experimental section}

Starting materials and solvents were purchased from commercial suppliers and were used without further purification. The progress of the reaction and purity of obtained compounds were controlled by TLC on "Kieselgel $60 \mathrm{~F}_{254}$ " plates (Merck). The structures of synthesized compounds were confirmed by $1 \mathrm{H}$ NMR spectrometry and liquid chromatography-mass spectra (LC-MS) analysis. Nuclear magnetic resonance spectra were recorded on a Varian Mercury VRX-400 spectrometer using
DMSO- $\mathrm{d}_{6}$ as solvent and tetramethylsilane as internal standard. Chemical shift values $(\delta)$ are quoted in ppm and coupling constants $(\mathrm{J})$ in Hz. Liquid chromatography-mass spectra (LC-MS) analyses were performed using the Agilent 1100 LC/MSD SL (Agilent Technologies) separations module and Mass Quad G1956B mass detector with electrospray ionization (Agilent Technologies). HPLC is performed using Zorbax SB-C18 (Agilent Technologies), Rapid Resolution HT Cartridge 4.6x30 mm 1.8- $\mu$ (Agilent Technologies $\mathrm{P} / \mathrm{N}: 823975-902)$ i.d. column, at a temperature of $40{ }^{\circ} \mathrm{C}$ with gradient elution of $0-100 \%$ $\mathrm{CH}_{3} \mathrm{CN}$ (with $1 \mathrm{~mL} / \mathrm{L} \mathrm{HCOOH}$ ): $\mathrm{H}_{2} \mathrm{O}$ (with $1 \mathrm{~mL} / \mathrm{L} \mathrm{HCOOH}$ ) at a flow rate of $3 \mathrm{~mL} / \mathrm{min}$ and a run time of $2.8 \mathrm{~min}$. Compounds were detected at $215 \mathrm{~nm}$ using a Diode Array G1315B detector. All tested compounds had $\geq 90 \%$ purity as determined by this method. All purified synthetic intermediates had $\geq 95 \%$ purity as determined by this method.

Method 1. A mixture of $107 \mathrm{~mL}$ (1 mol) of ethyl cyanoacetate, $32 \mathrm{~g}(1 \mathrm{~mol})$ of elemental sulfur and $1 \mathrm{~mol}$ of the corresponding ketone

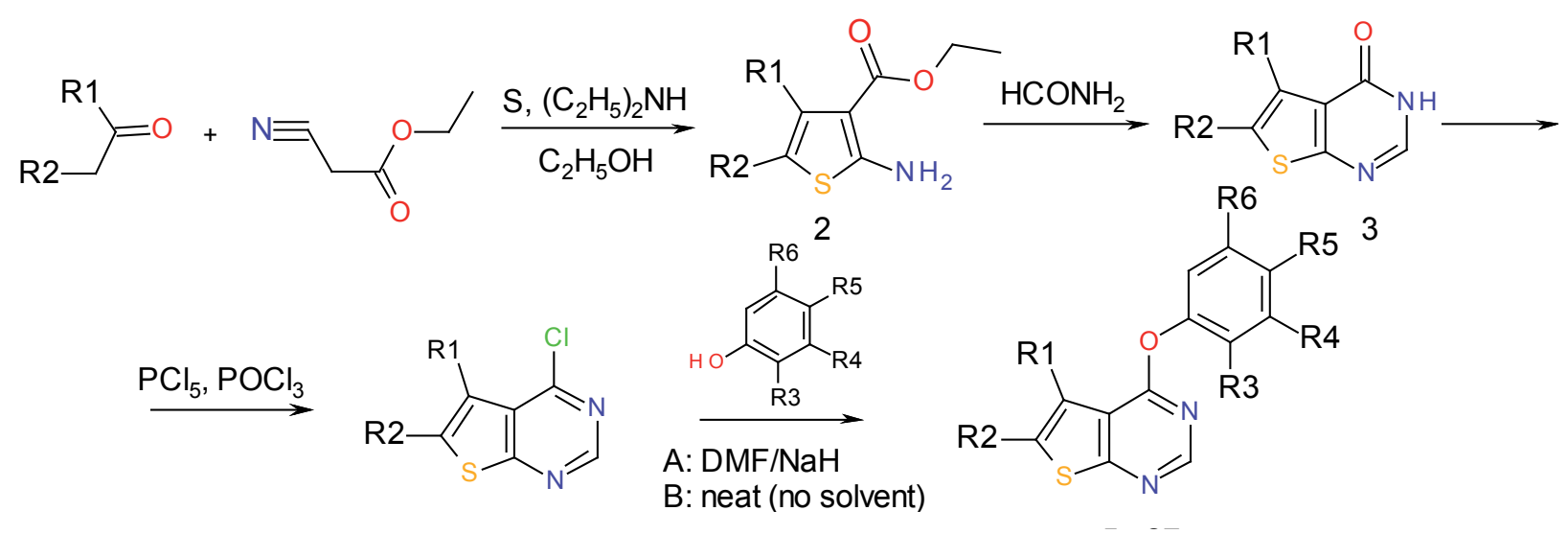

Scheme 1. General synthesis of compounds 5-27. 
in $200 \mathrm{ml}$ of ethanol was stirred at room temperature. $80 \mathrm{ml}$ of diethylamine were added to this mixture for $12 \mathrm{~h}$. The reaction mixture was left overnight; the precipitate was filtered, washed by aqueous alcohol (1:1) and dried in air [15]. Yield was 50-88\%.

Method 2. 2 mol of formamide were added to $1 \mathrm{~mol} 2$-aminothiophene and the mixture was heated to $160-170^{\circ} \mathrm{C}$ for $24 \mathrm{~h}$. The hot mixture was diluted with isopropyl alcohol and cooled. The solid product was collected by filtration, washed with isopropyl alcohol and water and dried at $60^{\circ} \mathrm{C}[16]$. Yield was $85-$ $90 \%$.

Method 3. A mixture of $0.01 \mathrm{~mol}$ of thienopyrimidinone, $11 \mathrm{ml}$ of $\mathrm{POCl}_{3}$ and $1.5 \mathrm{~g} \mathrm{PCl}_{5}$ was refluxed for 5-6 hours. The solvent was carefully evaporated to dryness. The dry product was dissolved in methylene chloride and poured to a cold $0.5 \mathrm{~N} \mathrm{NaOH}$ solution. The organic phase was separated and dried with anhydrous $\mathrm{Na}_{2} \mathrm{SO}_{4}$, then solvent was evaporated in vacuum. The residue was recrystallized from isopropanol [17]. Yield was 70$80 \%$.

Method 4A. NaH (60\% dispersion in mineral oil) $(0.5 \mathrm{~g}, 0.012 \mathrm{~mol})$ was added to solution of $0.011 \mathrm{~mol}$ of corresponding phenol in $10 \mathrm{ml}$ of dimethylformamide at $0^{\circ} \mathrm{C}$, after 30 minutes at ambient temperature $0.01 \mathrm{~mol}$ of substituted 4-chloro-thieno[2,3-d]pyrimidine was added and the mixture was stirred at $80^{\circ} \mathrm{C}$ for 8 hours. The content of the flask was diluted with water. Precipitate was filtered off and dried at room temperature. The resulting solid was recrystallized from ethanol. Yield was $60-80 \%$.

Method 4B. A mixture of $0.01 \mathrm{~mol} \mathrm{~N}-(4-$ hydroxy-phenyl)-acetamide (or N-(3-hydroxy- phenyl)-acetamide) and $0.01 \mathrm{~mol}$ of substituted 4-chloro-thieno[2,3-d]pyrimidine was melted at $180^{\circ} \mathrm{C}$ for $30 \mathrm{~min}$. Saturated sodium carbonate solution was added and the precipitate was filtered, washed with water and dried. The residue was recrystallized from mixture of N,N-dimethylformamide and ethanol (for compounds 6,10,12,23,24). Yield 50-60\%.

4-(4-methylphenoxy)-5,6,7,8-tetrahydro[1] benzothieno[2,3-d]pyrimidine (5). Yellow solid, m.p. $102-104^{\circ} \mathrm{C} ;{ }^{1} \mathrm{H}$ NMR (DMSO-d ${ }_{6}$ ) $\delta: 1.90\left(\mathrm{~s}, 4 \mathrm{H}, \mathrm{CH}_{2}\right), 2.35\left(\mathrm{~s}, 3 \mathrm{H}, \mathrm{CH}_{3}\right), 2.75$ (s, 2H, $\left.\mathrm{CH}_{2}\right), 3.05\left(\mathrm{~s}, 2 \mathrm{H}, \mathrm{CH}_{2}\right), 7.08(\mathrm{~d}, \mathrm{~J}=$ $\left.8.3 \mathrm{~Hz}, 2 \mathrm{H}, \mathrm{C}_{6} \mathrm{H}_{4}\right), 7.22(\mathrm{~d}, \mathrm{~J}=8.3 \mathrm{~Hz}, 2 \mathrm{H}$, $\left.\mathrm{C}_{6} \mathrm{H}_{4}\right), 8.37$ (s, $1 \mathrm{H}$, pyrimidine). LC-MS: $\mathrm{m} / z$ $297.0[\mathrm{M}+\mathrm{H}]^{+}$. Yield $65 \%$.

$N$-\{3-I(5-phenylthieno[2,3-d]pyrimidin4-yl)oxylphenylsacetamide (6). Brown solid, m.p. $190-191{ }^{\circ} \mathrm{C} ;{ }^{1} \mathrm{H}$ NMR (DMSO-d ${ }_{6}$ ) $\delta: 2.03$ $\left(\mathrm{s}, 3 \mathrm{H}, \mathrm{CH}_{3}\right), 6.82(\mathrm{~s}, 1 \mathrm{H}$, pyrimidine $), 7.28-7.5$ $\left(\mathrm{m}, 5 \mathrm{H}, \mathrm{C}_{6} \mathrm{H}_{5}\right), 7.62(\mathrm{~m}, 3 \mathrm{H}, \mathrm{Ar}), 7.8(\mathrm{~s}, 1 \mathrm{H}$, Ar), $8.6(\mathrm{~s}, 1 \mathrm{H}$, pyrimidine $), 9.92(\mathrm{~s}, 1 \mathrm{H}, \mathrm{NH})$. LC-MS: $m / z 362.0[\mathrm{M}+\mathrm{H}]^{+}$. Yield $50 \%$.

7-\{[5-(4-bromophenyl)thieno[2,3-d]pyrimidin-4-yl]oxy\}-2H-chromen-2-one (7). White solid, m.p. $216-217^{\circ} \mathrm{C} ;{ }^{1} \mathrm{H}$ NMR (DMSO-d ${ }_{6}$ ) $\delta: 6.43(\mathrm{~m}, 1 \mathrm{H}, \mathrm{CH}), 7.18(\mathrm{~m}, 1 \mathrm{H}, \mathrm{CH}), 7.36$ (s, $1 \mathrm{H}$, pyrimidine), $7.58(\mathrm{~m}, \mathrm{~J}=7.6 \mathrm{~Hz}, 2 \mathrm{H}$, $\left.\mathrm{C}_{6} \mathrm{H}_{4}\right), 7.63\left(\mathrm{~d}, \mathrm{~J}=7.6 \mathrm{~Hz}, 2 \mathrm{H}, \mathrm{C}_{6} \mathrm{H}_{4}\right), 7.75-$ $8.08(\mathrm{~m}, 3 \mathrm{H}), 8.63(\mathrm{~s}, 1 \mathrm{H}$, pyrimidine $)$, LCMS: $m / z 450.9[\mathrm{M}+\mathrm{H}]^{+}$. Yield $65 \%$.

4-(1,3-benzodioxol-5-yloxy)-5-(4-chlorophenyl)thieno[2,3-d]pyrimidine (8). Yellow solid, m.p. $156-158^{\circ} \mathrm{C} .{ }^{1} \mathrm{H}$ NMR (DMSO-d ${ }_{6}$ ) $\delta: 6.08\left(\mathrm{~s}, 2 \mathrm{H}, \mathrm{CH}_{2}\right), 6.7(\mathrm{~m}, 1 \mathrm{H}, \mathrm{Ar}), 6.92-7.06$ $(\mathrm{m}, 2 \mathrm{H}, \mathrm{Ar}), 7.52\left(\mathrm{~d}, \mathrm{~J}=7.6 \mathrm{~Hz}, 2 \mathrm{H}, \mathrm{C}_{6} \mathrm{H}_{4}\right)$, $7.72\left(\mathrm{~d}, \mathrm{~J}=7.6 \mathrm{~Hz}, 2 \mathrm{H}, \mathrm{C}_{6} \mathrm{H}_{4}\right), 7.94(\mathrm{~s}, 1 \mathrm{H}$, pyrimidine), 8.67 (s, $1 \mathrm{H}$, pyrimidine). LC-MS: $\mathrm{m} / \mathrm{z} 383.0[\mathrm{M}+\mathrm{H}]^{+}$. Yield $80 \%$. 
1-(4-\{[5-(4-methylphenyl)thieno[2,3-d] pyrimidin-4-yl]oxy?phenyl)ethanone (9). White solid, m.p. $134-135^{\circ} \mathrm{C}$; ${ }^{1} \mathrm{H}$ NMR $\left(\mathrm{DMSO}_{-} \mathrm{d}_{6}\right) \delta: 2.35\left(\mathrm{~s}, 3 \mathrm{H}, \mathrm{CH}_{3}\right), 2.58(\mathrm{~s}, 3 \mathrm{H}$, $\left.\mathrm{CH}_{3}\right), 7.2\left(\mathrm{~d}, \mathrm{~J}=7.8 \mathrm{~Hz}, 2 \mathrm{H}, \mathrm{C}_{6} \mathrm{H}_{4}\right), 7.29(\mathrm{~d}$, $\left.\mathrm{J}=7.8 \mathrm{~Hz}, 2 \mathrm{H}, \mathrm{C}_{6} \mathrm{H}_{4}\right), 7.53(\mathrm{~d}, \mathrm{~J}=7.6 \mathrm{~Hz}, 2 \mathrm{H}$, $\left.\mathrm{C}_{6} \mathrm{H}_{4}\right), 7.74(\mathrm{~s}, 1 \mathrm{H}$, pyrimidine), $8.02(\mathrm{~d}, \mathrm{~J}=$ $\left.7.6 \mathrm{~Hz}, 2 \mathrm{H}, \mathrm{C}_{6} \mathrm{H}_{4}\right), 8.57$ (s, $1 \mathrm{H}$, pyrimidine). LC-MS: $m / z 361.0[\mathrm{M}+\mathrm{H}]^{+}$. Yield $72 \%$.

$\mathrm{N}$-(4-\{[5-(4-methylphenyl)thieno[2,3-d] pyrimidin-4-yl]oxy?phenyl)acetamide (10). White solid, m.p. $204-205^{\circ} \mathrm{C} ;{ }^{1} \mathrm{H}$ NMR $\left(\mathrm{DMSO}_{6} \mathrm{~d}_{6}\right) \delta: 2.03\left(\mathrm{~s}, 3 \mathrm{H}, \mathrm{CH}_{3}\right), 2.33(\mathrm{~s}, 3 \mathrm{H}$, $\left.\mathrm{CH}_{3}\right), 7.04\left(\mathrm{~d}, \mathrm{~J}=7.6 \mathrm{~Hz}, 2 \mathrm{H}, \mathrm{C}_{6} \mathrm{H}_{4}\right), 7.21(\mathrm{~d}$, $\left.\mathrm{J}=6.3 \mathrm{~Hz}, 2 \mathrm{H}, \mathrm{C}_{6} \mathrm{H}_{4}\right), 7.52(\mathrm{~d}, \mathrm{~J}=6.3 \mathrm{~Hz}, 2 \mathrm{H}$, $\left.\mathrm{C}_{6} \mathrm{H}_{4}\right), 7.58\left(\mathrm{~d}, \mathrm{~J}=7.6 \mathrm{~Hz}, 2 \mathrm{H}, \mathrm{C}_{6} \mathrm{H}_{4}\right), 7.69(\mathrm{~s}$, $1 \mathrm{H}$, pyrimidine), 8.53 (s, $1 \mathrm{H}$, pyrimidine), 9.91 (s, $1 \mathrm{H}, \mathrm{NH})$. LC-MS: $m / z 376.0[\mathrm{M}+\mathrm{H}]^{+}$. Yield $58 \%$.

4-(3-chloro-4-fluorophenoxy)5-phenylthieno[2,3-d]pyrimidine (11). Blue solid, m.p. $143-145^{\circ} \mathrm{C} ;{ }^{1} \mathrm{H}$ NMR (DMSO-d $\left.\mathrm{d}_{6}\right)$ $\delta: ~ 7.16-7.2(\mathrm{~m}, 1 \mathrm{H}, \mathrm{Ar}), 7.33-7.49$ (m, 5H, Ar), 7.65 (d, 2H, Ar), 7.78 (s, 1H, pyrimidine), 8.57 (s, $1 \mathrm{H}$, pyrimidine). LC-MS: $\mathrm{m} / z \quad 357.0$ $[\mathrm{M}+\mathrm{H}]^{+}$. Yield $76 \%$.

$N-(3-\{[5-(4-m e t h o x y p h e n y l) t h i e n o[2,3-d]$ pyrimidin-4-yl]oxy\}phenyl)acetamide (12). Cream solid, m.p. $160-161^{\circ} \mathrm{C} ;{ }^{1} \mathrm{H}$ NMR $\left(\mathrm{DMSO}_{\mathrm{d}}\right.$ ) $\delta: 2.03\left(\mathrm{~s}, 3 \mathrm{H}, \mathrm{CH}_{3}\right), 3.77(\mathrm{~s}, 3 \mathrm{H}$, $\left.\mathrm{CH}_{3}\right), 6.96\left(\mathrm{~d}, \mathrm{~J}=8.1 \mathrm{~Hz}, 2 \mathrm{H}, \mathrm{C}_{6} \mathrm{H}_{4}\right), 7.28-7.37$ (m, 3H, Ar), 7.5 (s, 1H, Ar), 7.58 (d, J = 8.1 Hz, $\left.2 \mathrm{H}, \mathrm{C}_{6} \mathrm{H}_{4}\right), 7.78(\mathrm{~s}, 1 \mathrm{H}$, pyrimidine), $8.62(\mathrm{~s}$, $1 \mathrm{H}$, pyrimidine), 10.06 (s, 1H, NH). LC-MS: $\mathrm{m} / \mathrm{z} 392.0[\mathrm{M}+\mathrm{H}]^{+}$. Yield $58 \%$.

4-(1,3-benzodioxol-5-yloxy)-5-(4-methylphenyl)thieno[2,3-d]pyrimidine (13). White solid, m.p. $145-147^{\circ} \mathrm{C} ;{ }^{1} \mathrm{H}$ NMR (DMSO-d 6 ) $\delta: 2.31\left(\mathrm{~s}, 3 \mathrm{H}, \mathrm{CH}_{3}\right), 2.49\left(\mathrm{~s}, 2 \mathrm{H}, \mathrm{CH}_{2}\right), 6.05$ (s, 1H, Ar), 6.89-6.94 (m, 2H, Ar), $7.21(\mathrm{~d}, \mathrm{~J}=$ $\left.7.8 \mathrm{~Hz}, 2 \mathrm{H}, \mathrm{C}_{6} \mathrm{H}_{4}\right), 7.56(\mathrm{~d}, \mathrm{~J}=7.8 \mathrm{~Hz}, 2 \mathrm{H}$, $\left.\mathrm{C}_{6} \mathrm{H}_{4}\right), 7.79(\mathrm{~s}, 1 \mathrm{H}$, pyrimidine), $8.62(\mathrm{~s}, 1 \mathrm{H}$, pyrimidine). LC-MS: $m / z 363.0[\mathrm{M}+\mathrm{H}]^{+}$. Yield $73 \%$.

4-(quinolin-8-yloxy)-5,6,7,8-tetrahydro[1] benzothieno[2,3-d]pyrimidine (14). Yellow solid, m.p. $193-194^{\circ} \mathrm{C} ;{ }^{1} \mathrm{H}$ NMR (DMSO-d 6 ) $\delta: 1.93\left(\mathrm{~s}, 4 \mathrm{H}, \mathrm{CH}_{2}\right), 2.91\left(\mathrm{~s}, 4 \mathrm{H}, \mathrm{CH}_{2}\right), 7.48-$ $7.72(\mathrm{~m}, 3 \mathrm{H}, \mathrm{Ar}), 7.92(\mathrm{~d}, \mathrm{~J}=8.1 \mathrm{~Hz}, 1 \mathrm{H}, \mathrm{Ar}$, quinoline), 8.18(s, $1 \mathrm{H}, \mathrm{Ar}$, quinoline), 8.39 (d, $\mathrm{J}=8.1 \mathrm{~Hz}, 1 \mathrm{H}$, Ar, quinoline), $8.68(\mathrm{~s}, 1 \mathrm{H}$, pyrimidine). LC-MS: $m / z 334.0[\mathrm{M}+\mathrm{H}]^{+}$. Yield $60 \%$.

4-(4-bromophenoxy)-5,6,7,8-tetrahydro[1] benzothieno[2,3-d]pyrimidine (15). Cream solid, m.p. $90-91{ }^{\circ} \mathrm{C} ;{ }^{1} \mathrm{H}$ NMR (DMSO-d $\left.{ }_{6}\right) \delta$ : $1.86\left(\mathrm{~s}, 4 \mathrm{H}, \mathrm{CH}_{2}\right), 2.87\left(\mathrm{~s}, 2 \mathrm{H}, \mathrm{CH}_{2}\right), 3.00(\mathrm{~s}$, $\left.2 \mathrm{H}, \mathrm{CH}_{2}\right), 7.27\left(\mathrm{~d}, \mathrm{~J}=7.6 \mathrm{~Hz}, 2 \mathrm{H}, \mathrm{C}_{6} \mathrm{H}_{4}\right), 7.65$ $\left(\mathrm{d}, \mathrm{J}=7.6 \mathrm{~Hz}, 2 \mathrm{H}, \mathrm{C}_{6} \mathrm{H}_{4}\right), 8.47(\mathrm{~s}, 1 \mathrm{H}, \mathrm{py}-$ rimidine). LC-MS: $m / z 360.9[\mathrm{M}+\mathrm{H}]^{+}$. Yield $72 \%$.

4-(4-fluorophenoxy)-5,6,7,8-tetrahydro[1] benzothieno[2,3-d]pyrimidine (16). Cream solid, m.p. 89-91 ${ }^{\circ} \mathrm{C} ;{ }^{1} \mathrm{H}$ NMR (DMSO-d $\left.{ }_{6}\right) \delta$ : $1.78\left(\mathrm{~m}, 4 \mathrm{H}, \mathrm{CH}_{2}\right), 2.75\left(\mathrm{~s}, 2 \mathrm{H}, \mathrm{CH}_{2}\right), 3.08(\mathrm{~s}$, $\left.2 \mathrm{H}, \mathrm{CH}_{2}\right), 7.43\left(\mathrm{~m}, 4 \mathrm{H}, \mathrm{C}_{6} \mathrm{H}_{4}\right), 8.38(\mathrm{~s}, 1 \mathrm{H}$, pyrimidine). LC-MS: $m / z 301.0[\mathrm{M}+\mathrm{H}]^{+}$. Yield $75 \%$.

4-(3-methylphenoxy)-5,6,7,8-tetrahydro[1] benzothieno[2,3-d]pyrimidine (17). Brown solid, m.p. $95-96^{\circ} \mathrm{C} ;{ }^{1} \mathrm{H}$ NMR $\left(\mathrm{DMSO}_{6} \mathrm{~d}_{6}\right) \delta$ : 1.87-1.96 (m, 4H, $\left.\mathrm{CH}_{2}\right), 2.33\left(\mathrm{~s}, 3 \mathrm{H}, \mathrm{CH}_{3}\right), 2.75$ (s, $\left.2 \mathrm{H}, \mathrm{CH}_{2}\right), 3.00\left(\mathrm{~s}, 2 \mathrm{H}, \mathrm{CH}_{2}\right), 7.03-7.11$ (m, $3 \mathrm{H}, \mathrm{Ar}), 7.26(\mathrm{~m}, 1 \mathrm{H}, \mathrm{Ar}), 8.23(\mathrm{~s}, 1 \mathrm{H}$, pyrimidine). LC-MS: $m / z 297.0[\mathrm{M}+\mathrm{H}]^{+}$. Yield $65 \%$.

4 - (2, 4-dichlorophenoxy) - 5, 6 dimethylthieno[2,3-d]pyrimidine (18). White solid, m.p. $125^{\circ} \mathrm{C} ;{ }^{1} \mathrm{H}$ NMR (DMSO-d $\left.{ }_{6}\right) \delta$ : 
$2.53\left(\mathrm{~s}, 6 \mathrm{H}, \mathrm{CH}_{3}\right), 7.57$ (d, 2H, Ar), $7.84(\mathrm{~s}$, $1 \mathrm{H}, \mathrm{Ar}), 8.50$ (s, $1 \mathrm{H}$, pyrimidine). LC-MS: $\mathrm{m} / \mathrm{z}$ $324.9[\mathrm{M}+\mathrm{H}]^{+}$. Yield $62 \%$.

4-[3-(trifluoromethyl)phenoxy]-6,7,8,9tetrahydro-5H-cyclohepta[4,5]thieno[2,3-d] pyrimidine (19). White solid, m.p. $83-84^{\circ} \mathrm{C}$; ${ }^{1} \mathrm{H}$ NMR (DMSO-d $\left.\mathrm{d}_{6}\right) \delta: 1.72\left(\mathrm{~m}, 4 \mathrm{H}, \mathrm{CH}_{2}\right)$, $1.89\left(\mathrm{~m}, 2 \mathrm{H}, \mathrm{CH}_{2}\right), 2.96\left(\mathrm{~m}, 2 \mathrm{H}, \mathrm{CH}_{2}\right), 3.25$ (m, 2H, $\left.\mathrm{CH}_{2}\right), 7.59-7.78(\mathrm{~m}, 4 \mathrm{H}, \mathrm{Ar}), 8.48(\mathrm{~s}$, 1H, pyrimidine). LC-MS: $m / z 365.0[\mathrm{M}+\mathrm{H}]^{+}$. Yield $71 \%$.

dimethyl 5-\{[5-(4-methylphenyl)thieno[2,3d]pyrimidin-4-yl]oxy\}isophthalate (20). Light brown solid, m.p. $103-105^{\circ} \mathrm{C} ;{ }^{1} \mathrm{H}$ NMR $\left(\mathrm{DMSO}_{6} \mathrm{~d}_{6}\right) \delta: 2.33\left(\mathrm{~s}, 3 \mathrm{H}, \mathrm{CH}_{3}\right), 3.12(\mathrm{~s}, 3 \mathrm{H}$, $\left.\mathrm{CH}_{3}\right), 3.89$ (s, $\left.3 \mathrm{H}, \mathrm{CH}_{3}\right), 7.32(\mathrm{~d}, \mathrm{~J}=7.8 \mathrm{~Hz}$, $\left.2 \mathrm{H}, \mathrm{C}_{6} \mathrm{H}_{4}\right), 7.58\left(\mathrm{~d}, \mathrm{~J}=7.8 \mathrm{~Hz}, 2 \mathrm{H}, \mathrm{C}_{6} \mathrm{H}_{4}\right), 7.76$ (s, 1H, Ar), 7.98 (s, 2H, Ar), 8.39 (s, 1H, pyrimidine), $8.60(\mathrm{~s}, 1 \mathrm{H}$, pyrimidine). LC-MS: $m / z 435.0[\mathrm{M}+\mathrm{H}]^{+}$. Yield $61 \%$.

7-[(5,6-dimethylthieno[2,3-d]pyrimidin-4-yl) oxyl-4-methyl-2H-chromen-2-one (21). White solid, m.p. $275-276^{\circ} \mathrm{C} ;{ }^{1} \mathrm{H}$ NMR $\left(\mathrm{DMSO}^{\left.-\mathrm{d}_{6}\right)} \delta\right.$ : $2.58\left(\mathrm{~s}, 6 \mathrm{H}, \mathrm{CH}_{3}\right), 3.05\left(\mathrm{~s}, 3 \mathrm{H}, \mathrm{CH}_{3}\right), 6.28(\mathrm{~s}$, $1 \mathrm{H}, \mathrm{CH}), 7.28(\mathrm{~m}, 1 \mathrm{H}, \mathrm{Ar}), 7.32(\mathrm{~m}, 1 \mathrm{H}, \mathrm{Ar})$, 7.80 (m, 1H, Ar) 8.45 (s, 1H, pyrimidine). LCMS: $m / z 339.0[\mathrm{M}+\mathrm{H}]^{+}$. Yield $72 \%$.

5-phenyl-4-[3-(trifluoromethyl)phenoxy] thieno[2,3-d]pyrimidine (22). White solid, m.p. $124-126^{\circ} \mathrm{C} ;{ }^{1} \mathrm{H}$ NMR (DMSO-d 6 ) $\delta$ : 7.34$7.50(\mathrm{~m}, 3 \mathrm{H}, \mathrm{Ar}), 7.60$ (s, 1H, Ar), 7.63-7.70 ( $\left.\mathrm{m}, 5 \mathrm{H}, \mathrm{C}_{6} \mathrm{H}_{5}\right), 7.83$ (s, $1 \mathrm{H}$, pyrimidine), 8.61 (s, $1 \mathrm{H}$, pyrimidine). LC-MS: $\mathrm{m} / \mathrm{z} 373.0$ $[\mathrm{M}+\mathrm{H}]^{+}$. Yield $75 \%$.

$N$-\{4-[(5,6-dimethylthieno[2,3-d]pyrimidin4-yl)oxylphenyl\}acetamide (23). White solid, m.p. $202-203^{\circ} \mathrm{C} ;{ }^{1} \mathrm{H}$ NMR (DMSO-d ${ }_{6}$ ) $\delta: 2.07$ $\left(\mathrm{s}, 3 \mathrm{H}, \mathrm{CH}_{3}\right), 2.57\left(\mathrm{~s}, 3 \mathrm{H}, \mathrm{CH}_{3}\right), 3.11(\mathrm{~s}, 3 \mathrm{H}$, $\left.\mathrm{CH}_{3}\right), 7.08\left(\mathrm{~d}, \mathrm{~J}=7.6 \mathrm{~Hz}, 2 \mathrm{H}, \mathrm{C}_{6} \mathrm{H}_{4}\right), 7.62(\mathrm{~d}$,
$\left.\mathrm{J}=7.6 \mathrm{~Hz}, 2 \mathrm{H}, \mathrm{C}_{6} \mathrm{H}_{4}\right), 8.37(\mathrm{~s}, 1 \mathrm{H}$, pyrimidine), 9.87 ( $\mathrm{s}, 1 \mathrm{H}, \mathrm{NH})$. LC-MS: $m / z 314.0$ $[\mathrm{M}+\mathrm{H}]^{+}$. Yield $56 \%$.

N-[3-(6,7-dihydro-5H-cyclopenta[4,5] thieno[2,3-d]pyrimidin-4-yloxy)phenyl]acetamide (24). Grey solid, m.p. $197-198^{\circ} \mathrm{C} ;{ }^{1} \mathrm{H}$ NMR (DMSO-d $\left.\mathrm{d}_{6}\right) \delta: 2.03\left(\mathrm{~s}, 3 \mathrm{H}, \mathrm{CH}_{3}\right), 2.43-$ $2.58\left(\mathrm{~m}, 2 \mathrm{H}, \mathrm{CH}_{2}\right), 3.03-3.18\left(\mathrm{~m}, 4 \mathrm{H}, \mathrm{CH}_{2}\right)$, $6.77(\mathrm{~d}, 1 \mathrm{H}, \mathrm{Ar}), 7.23(\mathrm{~m}, 1 \mathrm{H}, \mathrm{Ar}), 7.38(\mathrm{~d}$, $1 \mathrm{H}, \mathrm{Ar}), 7.56$ (s, 1H, Ar), 8.33 (s, 1H, pyrimidine), 9.78 (s, 1H, NH). LC-MS: $m / z 326.0$ $[\mathrm{M}+\mathrm{H}]^{+}$. Yield $53 \%$.

6-methyl-5-phenyl-4-[3-(trifluoromethyl) phenoxy]thieno[2,3-d]pyrimidine (25). White solid, m.p. $161-163^{\circ} \mathrm{C} ;{ }^{1} \mathrm{H}$ NMR (DMSO-d ${ }_{6}$ ) $\delta: 2.83\left(\mathrm{~s}, 3 \mathrm{H}, \mathrm{CH}_{3}\right), 7.21-7.53(\mathrm{~m}, 9 \mathrm{H}, \mathrm{Ar})$, 8.43 (s, 1H, pyrimidine). LC-MS: $m / z 387.0$ $[\mathrm{M}+\mathrm{H}]^{+}$. Yield $78 \%$.

Methyl 4-[(6-methyl-5-phenylthieno[2,3-d] pyrimidin-4-yl)oxy]benzoate (26). Grey solid, m.p. $147-148^{\circ} \mathrm{C} ;{ }^{1} \mathrm{H}$ NMR (DMSO-d ${ }_{6}$ ) $\delta: 3.20$ $\left(\mathrm{s}, 3 \mathrm{H}, \mathrm{CH}_{3}\right), 3.87\left(\mathrm{~s}, 3 \mathrm{H}, \mathrm{CH}_{3}\right), 7.17(\mathrm{~d}, \mathrm{~J}=$ $\left.7.8 \mathrm{~Hz}, 2 \mathrm{H}, \mathrm{C}_{6} \mathrm{H}_{4}\right), 7.33-7.58\left(\mathrm{~m}, 5 \mathrm{H}, \mathrm{C}_{6} \mathrm{H}_{5}\right) 7.96$ $\left(\mathrm{d}, \mathrm{J}=7.8 \mathrm{~Hz}, 2 \mathrm{H}, \mathrm{C}_{6} \mathrm{H}_{4}\right), 8.53$ (s, $1 \mathrm{H}$, pyrimidine). LC-MS: $m / z 377.0[\mathrm{M}+\mathrm{H}]^{+}$. Yield $69 \%$.

7-methyl-4-[3-(trifluoromethyl)phenoxy]5,6,7,8-tetrahydro[1]benzothieno[2,3-d]pyrimidine (27). Yellow solid, m.p. $95-96^{\circ} \mathrm{C} ;{ }^{1} \mathrm{H}$ NMR (DMSO-d $\left.\mathrm{d}_{6}\right) \delta: 1.11\left(\mathrm{~d}, 3 \mathrm{H}, \mathrm{CH}_{3}\right), 1.40-$ $1.58(\mathrm{~m}, 1 \mathrm{H}, \mathrm{CH}), 1.88-2.04\left(\mathrm{~m}, 2 \mathrm{H}, \mathrm{CH}_{2}\right)$, 2.88-3.02 (m, $\left.2 \mathrm{H}, \mathrm{CH}_{2}\right), 3.15-3.23(\mathrm{~m}, 2 \mathrm{H}$, $\mathrm{CH}_{2}$ ), 7.62-7.78 (m, 4H, Ar), 8.51 (s, 1H, pyrimidine). LC-MS: $m / z 365.0[\mathrm{M}+\mathrm{H}]^{+}$. Yield $69 \%$.

\section{Results and Discussion}

Previously N-phenylthieno[2,3-d]pyrimidin4-amines were identified as FGFR1 inhibitors [6]. In this study 4-methoxythieno[2,3-d]py- 
Table 1. Structure, in vitro inhibitory activity toward FGFR1 and $\mathbf{I C}_{\mathbf{5 0}}$ of studied compounds.<smiles>[R]c1cc(Oc2ncnc3sc([R])c([R])c23)c([R])c([R])c1[R]</smiles>

\begin{tabular}{|c|c|c|c|c|c|c|c|c|c|}
\hline \multirow{2}{*}{$\begin{array}{l}\text { Com- } \\
\text { pound }\end{array}$} & \multirow[t]{2}{*}{$\mathrm{R}^{1}$} & \multirow[t]{2}{*}{$\mathrm{R}^{2}$} & \multirow[t]{2}{*}{$\mathrm{R}^{3}$} & \multirow[t]{2}{*}{$\mathrm{R}^{4}$} & \multirow[t]{2}{*}{$\mathrm{R}^{5}$} & \multirow[t]{2}{*}{$\mathrm{R}^{6}$} & \multicolumn{2}{|c|}{$\begin{array}{l}\text { Residual activity of } \\
\text { FGFR1 at, \% }\end{array}$} & \multirow[t]{2}{*}{$\mathrm{IC}_{50}, \mu \mathrm{M}$} \\
\hline & & & & & & & $33 \mu \mathrm{M}$ & $10 \mu \mathrm{M}$ & \\
\hline 5 & \multicolumn{2}{|l|}{$-\left(\mathrm{CH}_{2}\right)_{4^{-}}$} & $\mathrm{H}$ & $\mathrm{H}$ & $\mathrm{Me}$ & $\mathrm{H}$ & 33.8 & 41.7 & 4.47 \\
\hline 6 & phenyl & $\mathrm{H}$ & $\mathrm{H}$ & $\mathrm{H}$ & NAc & $\mathrm{H}$ & 24.5 & $\mathrm{~N} / \mathrm{A}$ & 2.3 \\
\hline 7 & 4-bromophenyl & $\mathrm{H}$ & $\mathrm{H}$ & & & $\mathrm{H}$ & 14.7 & 40.3 & 2.45 \\
\hline 8 & 4-chlorophenyl & $\mathrm{H}$ & $\mathrm{H}$ & & & $\mathrm{H}$ & 29.6 & 30.1 & 2.14 \\
\hline 9 & 4-methylphenyl & $\mathrm{H}$ & $\mathrm{H}$ & $\mathrm{H}$ & Ac & $\mathrm{H}$ & 26.9 & $\mathrm{~N} / \mathrm{A}$ & 5 \\
\hline 10 & 4-methylphenyl & $\mathrm{H}$ & $\mathrm{H}$ & $\mathrm{H}$ & NAc & $\mathrm{H}$ & 27.7 & $\mathrm{~N} / \mathrm{A}$ & 2.5 \\
\hline 11 & phenyl & $\mathrm{H}$ & $\mathrm{H}$ & $\mathrm{Cl}$ & $\mathrm{F}$ & $\mathrm{H}$ & 21.8 & 33,1 & 3.55 \\
\hline 12 & 4-methoxyphenyl & $\mathrm{H}$ & $\mathrm{H}$ & $\mathrm{H}$ & NAc & $\mathrm{H}$ & 19.3 & $\mathrm{~N} / \mathrm{A}$ & 5.6 \\
\hline 13 & 4-methylphenyl & $\mathrm{H}$ & $\mathrm{H}$ & & & $\mathrm{H}$ & 15.1 & 17.4 & 0.9 \\
\hline 14 & \multicolumn{2}{|l|}{$-\left(\mathrm{CH}_{2}\right)_{4^{-}}$} & \multicolumn{2}{|c|}{$\begin{array}{l}\cdots \\
\cdots N\end{array}$} & $\mathrm{H}$ & $\mathrm{H}$ & N/A & 86,5 & N/A \\
\hline 15 & \multicolumn{2}{|l|}{$-\left(\mathrm{CH}_{2}\right)_{4^{-}}$} & $\mathrm{H}$ & $\mathrm{H}$ & $\mathrm{Br}$ & $\mathrm{H}$ & 52 & $\mathrm{~N} / \mathrm{A}$ & $\mathrm{N} / \mathrm{A}$ \\
\hline 16 & \multicolumn{2}{|l|}{$-\left(\mathrm{CH}_{2}\right)_{4^{-}}$} & $\mathrm{H}$ & $\mathrm{H}$ & $\mathrm{F}$ & $\mathrm{H}$ & 50.4 & $\mathrm{~N} / \mathrm{A}$ & N/A \\
\hline 17 & \multicolumn{2}{|l|}{$-\left(\mathrm{CH}_{2}\right)_{4^{-}}$} & $\mathrm{H}$ & $\mathrm{Me}$ & $\mathrm{H}$ & $\mathrm{H}$ & 65.3 & $\mathrm{~N} / \mathrm{A}$ & N/A \\
\hline 18 & $\mathrm{Me}$ & $\mathrm{Me}$ & $\mathrm{Cl}$ & $\mathrm{H}$ & $\mathrm{Cl}$ & $\mathrm{H}$ & 88.2 & $\mathrm{~N} / \mathrm{A}$ & $\mathrm{N} / \mathrm{A}$ \\
\hline 19 & \multicolumn{2}{|l|}{$-\left(\mathrm{CH}_{2}\right)_{5^{-}}$} & $\mathrm{H}$ & $\mathrm{CF}_{3}$ & $\mathrm{H}$ & $\mathrm{H}$ & 64.3 & $\mathrm{~N} / \mathrm{A}$ & N/A \\
\hline 20 & 4-methylphenyl & $\mathrm{H}$ & $\mathrm{H}$ & COOMe & $\mathrm{H}$ & COOMe & 53.2 & 83.7 & $\mathrm{~N} / \mathrm{A}$ \\
\hline
\end{tabular}


Ended Table 1

\begin{tabular}{|c|c|c|c|c|c|c|c|c|c|}
\hline \multirow{2}{*}{$\begin{array}{l}\text { Com- } \\
\text { pound }\end{array}$} & \multirow{2}{*}{$\mathrm{R}^{1}$} & \multirow{2}{*}{$\mathrm{R}^{2}$} & \multirow[t]{2}{*}{$\mathrm{R}^{3}$} & \multirow[t]{2}{*}{$\mathrm{R}^{4}$} & \multirow[t]{2}{*}{$\mathrm{R}^{5}$} & \multirow[t]{2}{*}{$\mathrm{R}^{6}$} & \multicolumn{2}{|c|}{$\begin{array}{c}\text { Residual activity of } \\
\text { FGFR1 at, } \% \\
\end{array}$} & \multirow{2}{*}{$\mathrm{IC}_{50}, \mu \mathrm{M}$} \\
\hline & & & & & & & $33 \mu \mathrm{M}$ & $10 \mu \mathrm{M}$ & \\
\hline 21 & $\mathrm{Me}$ & $\mathrm{Me}$ & $\mathrm{H}$ & & & $\mathrm{H}$ & N/A & N/A & N/A \\
\hline 22 & phenyl & $\mathrm{H}$ & $\mathrm{H}$ & $\mathrm{CF}_{3}$ & $\mathrm{H}$ & $\mathrm{H}$ & 70.4 & N/A & N/A \\
\hline 23 & $\mathrm{Me}$ & $\mathrm{Me}$ & $\mathrm{H}$ & $\mathrm{H}$ & NAc & $\mathrm{H}$ & 65.8 & $\mathrm{~N} / \mathrm{A}$ & $\mathrm{N} / \mathrm{A}$ \\
\hline 24 & & & $\mathrm{H}$ & NAc & $\mathrm{H}$ & $\mathrm{H}$ & N/A & $\mathrm{N} / \mathrm{A}$ & $\mathrm{N} / \mathrm{A}$ \\
\hline 25 & phenyl & $\mathrm{Me}$ & $\mathrm{H}$ & $\mathrm{CF}_{3}$ & $\mathrm{H}$ & $\mathrm{H}$ & 53.5 & 90.5 & N/A \\
\hline 26 & phenyl & $\mathrm{Me}$ & $\mathrm{H}$ & $\mathrm{H}$ & COOMe & $\mathrm{H}$ & 42.7 & 50 & N/A \\
\hline 27 & & & $\mathrm{H}$ & $\mathrm{CF}_{3}$ & $\mathrm{H}$ & $\mathrm{H}$ & 80 & $\mathrm{~N} / \mathrm{A}$ & N/A \\
\hline
\end{tabular}

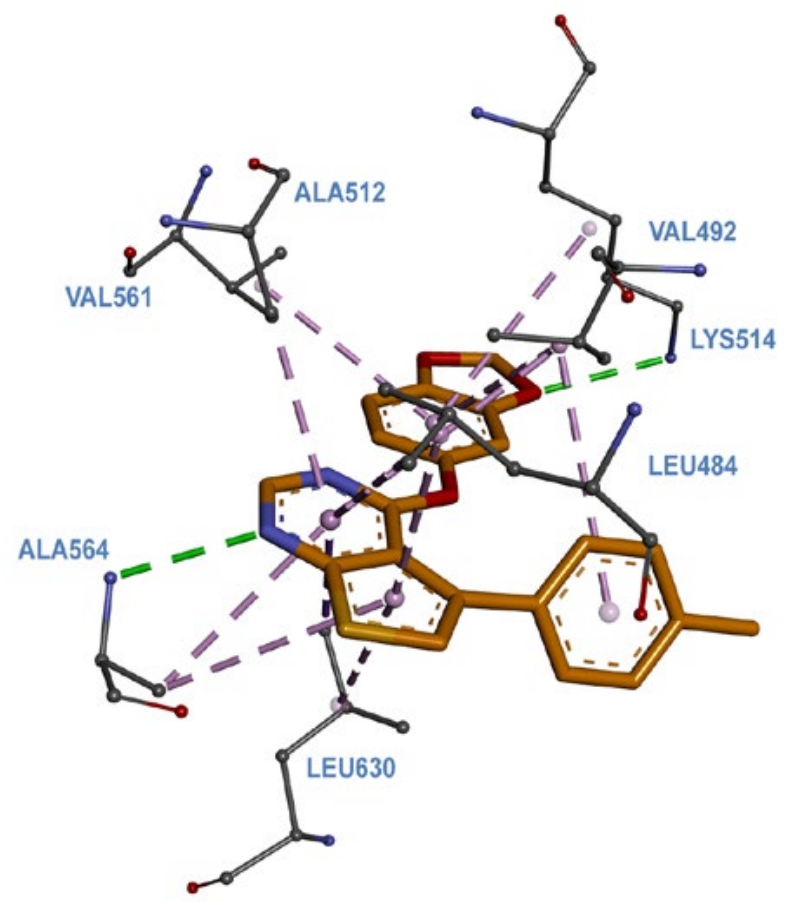

Fig. 1. The complex of compound $\mathbf{1 3}$ with ATP-acceptor site of FGFR1 obtained with Autodock 4.2.6. H-bonds are shown by green dashed lines, hydrophobic interactions - purple dashed lines. rimidines have been synthesized and tested toward FGFR1 kinases.

Twenty three 4-methoxythieno[2,3-d]pyrimidines were evaluated for ability to inhibit the FGFR1 activity using ${ }^{32} \mathrm{P}-\mathrm{ATP}$ radiolabeled assay. This method provides direct measurement of the enzyme activity. The results are summarized in Table 1. Nine compounds showed inhibitory activity with $\mathrm{IC}_{50}$ value from 0.9 to $5.6 \mu \mathrm{M}$.

To understand the activity of the compounds and make some conclusions about SAR we have performed molecular docking and analyzed the complexes of studied compounds with FGFR1 ATP-binding pocket to propose their binding mode. The complex of the most active found compound with the ATP-acceptor site of FGFR1 is shown in Fig. 1.

Proposed binding mode of the studied compounds belongs to the type I protein kinases inhibitors. The thieno[2,3-d]pyrimidine core is located in adenine binding region and forms 
hydrogen bond with the amino acid residue Ala564 of the hinge region. Most $\mathrm{R}^{3}-\mathrm{R}^{6}$ substituents are extended towards hydrophobic region I and form hydrophobic interactions with Ala512 and Val561 and hydrogen bonds with Lys514 or Asp641. R ${ }^{1}-\mathrm{R}^{2}$ substituents are oriented to hydrophobic region II and form hydrophobic interactions with Val492 and/or Leu630 and/or Leu484. As exceptions were the compounds 14, 15, 16, 19 and 24 with 5and 6-membered cycles at $\mathrm{R}^{1}-\mathrm{R}^{2} \cdot \mathrm{R}^{1}-\mathrm{R}^{2}$ substituents of these compounds are oriented to the hydrophobic region I and $\mathrm{R}^{3}-\mathrm{R}^{6}$ substituents are oriented to the outer of binding site.

The Structure Activity Relationship (SAR) study showed that $\mathrm{R}^{1}$ of the most active compounds is phenyl. Mostly it is a hydrophobic 4-substituted phenyl. The compound $\mathbf{1 0}$ with 4-methylphenyl (more hydrophobic) at $\mathrm{R}^{1}$ has two-fold higher activity than compound $\mathbf{1 2}$ with 4-methoxyphenyl at $\mathrm{R}^{1}$. $\mathrm{IC}_{50}$ values were 2.5 and $5.6 \mu \mathrm{M}$ respectively. But the compound $\mathbf{8}$ with 4-chlorophenyl (more hydrophobic) at $\mathrm{R}^{1}$ has lower activity than compound 13 with 4-methylphenyl. $\mathrm{IC}_{50}$ values were 2.14 and $0.9 \mu \mathrm{M}$, respectively. Therefore, the order of potency for the substituents at $\mathrm{R}^{1}$ could be proposed as following: 4-methylphenyl $>4$-chlorophenyl >4-methoxyphenyl.

SAR study of substituents at $\mathrm{R}^{3}-\mathrm{R}^{6}$ showed that the most active compounds have hydrogen bond acceptor at positions $\mathrm{R}^{4}$ and/or $\mathrm{R}^{5}$. Compounds 8 and $\mathbf{1 3}$ contain dioxymethan and have $\mathrm{IC}_{50}$ values of 2.14 and $0.9 \mu \mathrm{M}$, respectively.

\section{Conclusions}

Nine new inhibitors of FGFR1 were discovered among 23 4-methoxythieno[2,3-d]py- rimidine derivatives. SAR study showed that the most profitable substituent at $\mathrm{R}^{1}$ is 4-methylphenyl and hydrogen bond acceptors at $\mathrm{R}^{4}$ and/or $\mathrm{R}^{5}$ are important for revealing the inhibitory activity toward FGFR1. The most potent compound in this series showed the $\mathrm{IC}_{50}$ value of $0.9 \mu \mathrm{M}$. The studied compounds are good candidates for the further development of effective FGFR1 inhibitors and potential anti-cancer drugs.

\section{Funding}

This work was supported by a grant from the Project of the National Academy of Sciences (NAS) of Ukraine № 0117U000204 "New approaches for target-oriented search of potential antitumor and anti-infectious agents to overcome the problem of drug resistance".

\section{REFERENCES}

1. Yu T, Yang Y, Liu Y, Zhang Y, Xu H, Li M, Ponnusamy $M$, Wang $K$, Wang $J X$, Li PF. A FGFR1 inhibitor patent review: progress since 2010. Expert Opin Ther Pat. 2017;27(4):439-454.

2. Chioni AM, Grose R. FGFR1 cleavage and nuclear translocation regulates breast cancer cell behavior. J Cell Biol. 2012;197(6):801-17.

3. Weiss J, Sos ML, Seidel D, Peifer M, Zander T, Heuckmann JM, Ullrich RT, Menon R, Maier $S$, Soltermann A, Moch H, Wagener P, Fischer F, Heynck S, Koker M, Schöttle J, Leenders F, Gabler F, Dabow I, Querings S, Heukamp LC, BalkeWant H, Ansén S, Rauh D, Baessmann I, Altmüller J, Wainer Z, Conron M, Wright G, Russell P, Solomon B, Brambilla E, Brambilla C, Lorimier P, Sollberg $S$, Brustugun OT, Engel-Riedel W, Ludwig C, Petersen I, Sänger J, Clement J, Groen H, Timens W, Sietsma H, Thunnissen E, Smit E, Heideman D, Cappuzzo F, Ligorio C, Damiani S, Hallek M, Beroukhim R, Pao W, Klebl B, Baumann M, Buettner R, Ernestus K, Stoelben E, Wolf J, Nürnberg P, Per- 
ner $S$, Thomas RK. Frequent and focal FGFR1 amplification associates with therapeutically tractable FGFR1 dependency in squamous cell lung cancer. Sci Transl Med. 2010;2(62):62ra93.

4. Sahores A, May M, Sequeira GR, Fuentes C, Jacobsen $B$, Lanari $C$, Lamb $C A$. Targeting FGFR with BGJ398 in Breast Cancer: Effect on Tumor Growth and Metastasis. Curr Cancer Drug Targets. 2018;18(10):979-987.

5. Gryshchenko AA, Levchenko $K V$, Bdzhola $V G$, Ruban TP, Lukash LL, Yarmoluk SM. Design, synthesis and biological evaluation of naphthostyril derivatives as novel protein kinase FGFR1 inhibitors. J Enzyme Inhib Med Chem. 2015;30(1): 126-32.

6. Gryshchenko AA, Bdzhola VG, Balanda AO, Briukhovetska NV, Kotey IM, Golub AG, Ruban TP, Lukash LL, Yarmoluk SM. Design, synthesis and biological evaluation of N-phenylthieno[2,3-d]pyrimidin-4-amines as inhibitors of FGFR1. Bioorg Med Chem. 2015;23(9):2287-93.

7. GGryshchenko AA, Bdzhola VG, Pletnyova LV, Chepurna RV, Zhitnetsky IV, Yarmoluk SM. Quinazolone inhibitors of protein kinase FGFR1. Ukr Bioorg Acta 2010; 8(2):63-8.

8. Gryshchenko AA, Bdzhola VG, Borovikov OV, Kukharenko OP, Pletnyova LV, Yarmoluk SM. Search for FGFR1 inhibitors among oxindole derivatives. Ukr Bioorg Acta; 2009; 7(2):64-8

9. Gryshchenko AA, Tarnavskiy SS, Levchenko KV, Bdzhola VG, Volynets GP, Golub AG, Ruban TP, Vygranenko KV, Lukash LL, Yarmoluk SM. Design, synthesis and biological evaluation of 5-amino4-(1H-benzoimidazol-2-yl)-phenyl-1,2-dihydropyrrol-3-ones as inhibitors of protein kinase FGFR1. Bioorg Med Chem. 2016;24(9):2053-9.

10. Grischenko AA, Bdzhola VG, Kukharenko OP, Yarmoluk SM. Search for FGFR1 inhibitors among chromone derivatives. Ukr Bioorg Acta; 2009; 7(1):50-6.

11. Morris GM, Huey R, Lindstrom W, Sanner MF, Belew RK, Goodsell DS, Olson AJ. AutoDock4 and AutoDockTools4: Automated docking with selective receptor flexibility. J Comput Chem. 2009;30(16): 2785-91.
12. Pedretti A, Villa L, Vistoli G. VEGA--an open platform to develop chemo-bio-informatics applications, using plug-in architecture and script programming. J Comput Aided Mol Des. 2004;18(3):167-73.

13. Syniugin AR, Ostrynska OV, Chekanov MO, Volynets GP, Starosyla SA, Bdzhola VG, Yarmoluk SM. Design, synthesis and evaluation of 3-quinoline carboxylic acids as new inhibitors of protein kinase CK2. J Enzyme Inhib Med Chem. 2016;31(sup4):160-9.

14. Bae JH, Lew ED, Yuzawa S, Tomé F, Lax I, Schlessinger $J$. The selectivity of receptor tyrosine kinase signaling is controlled by a secondary $\mathrm{SH} 2$ domain binding site. Cell. 2009;138(3):514-24.

15. Sabnis $R W$, Rangnekar DW, Sonawane ND. 2-aminothiophenes by the gewald reaction. $J$ Heterocycl Chem. 1999;36(2):333-45.

16. Golub AG, Bdzhola VG, Briukhovetska NV, Balanda AO, Kukharenko OP, Kotey IM, Ostrynska OV, Yarmoluk SM. Synthesis and biological evaluation of substituted (thieno[2,3-d]pyrimidin-4-ylthio)carboxylic acids as inhibitors of human protein kinase CK2. Eur J Med Chem. 2011;46(3):870-6.

17. Ostrynska OV, Balanda AO, Bdzhola VG, Golub AG, Kotey IM, Kukharenko OP, Gryshchenko AA, Briukhovetska NV, Yarmoluk SM. Design and synthesis of novel protein kinase CK2 inhibitors on the base of 4-aminothieno[2,3-d]pyrimidines. Eur J Med Chem. 2016;115:148-60.

\section{Ідентифікація інгібіторів FGFR1 серед 4-метокситієно[2,3-d]піримідинів.}

I. М. Котей, М. В. Протопопов, С. А. Старосила, А. О. Баланда, Л. В. Плетньова, А. О. Приходько, В. Г. Бджола та С. М. Ярмолюк

Мета. Пошук нових інгібіторів протеїнкінази FGFR1. Методи. В роботі використовувались методи органічного синтезу, молекулярний докінг програмним пакетом Autodock 4.2.6 та біохімічне тестування in vitro із використанням $\gamma_{-}{ }^{32} \mathrm{P}$ АТФ. Результати. Експерименти in vitro показали, що 9 з 23 сполук проявляють інгібувальну активність щодо FGFR1 зі значенням $\mathrm{IC}_{50}$ в межах від 0.9 до $5.6 \mu \mathrm{M}$. Висновки. Було розроблено дев'ять інгібіторів FGFR1. За допомогою методів молекулярного моделювання було визначено спосіб зв'язування 
iз АТФ-акцепторним сайтом та вивченозалежність активності сполук від замісників $\mathrm{R}^{1}, \mathrm{R}^{4}$ та $\mathrm{R}^{5}$.

Кл ю ч о в і с л о в а. Протеїнкіназа FGFR1, молекулярний докінг, органічний синтез, in vitro тестування.

\section{Идентификация ингибиторов FGFR1 среди 4-метокситиено[2,3-d]пиримидинов}

И. М. Котей, Н. В. Протопопов, С. А. Старосила, Л. В. Плетнёва, А. А. Приходько, А. А. Баланда, В. Г. Бджола и С. М. Ярмолюк

Цель. Поиск новых химических соединений со способностью ингибировать протеинкиназу FGFR1. Методы. В работе использовались методы органического синтеза, молекулярного докинга программным пакетом Autodock 4.2.6 и биохимическое тестирование in vitro, используя $\gamma$-32P АТФ. Результаты. Биохимическое тестирование показало, что 9 из 23 исследуемых соединений ингибируют протеинкиназу FGFR1 в диапазоне значений $\mathrm{IC}_{50}$ от 0.9 до $5.6 \mu \mathrm{M}$. Выводы. Было разработано девять ингибиторов FGFR1. C помощью молекулярного докинга было определено положение лигандов в АТФ-акцепторном сайте и изучено зависимость активности от структуры заместителей $\mathrm{R}^{1}, \mathrm{R}^{4}$ та $\mathrm{R}^{5}$.

К л юч е в ы е с л о в а: Протеинкиназа FGFR1, молекулярный докинг, органический синтез, in vitro тестирование, 4-метокситиено[2,3-d]пиримидин.

Received 10.10.2018 